Check for updates

Cite this: RSC Adv., 2017, 7, 50150

Received 20th July 2017

Accepted 21st September 2017

DOI: $10.1039 / \mathrm{c} 7 \mathrm{ra07997d}$

rsc.li/rsc-advances

\section{The diversities of magnetic behaviors appearing simultaneously in three isomorphs based on $1 \mathrm{H}$ - tetrazolyl-acetic acid ligand $\uparrow$}

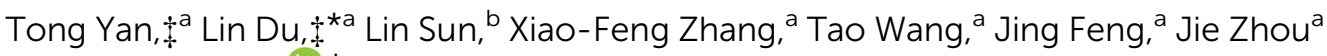 \\ and Qi-Hua Zhao (D)*a
}

\begin{abstract}
Three isomorphous metal-organic complexes with $1 \mathrm{H}$-tetrazolyl-acetic acid (TAA), namely $\left[M(T A A)_{2}\right]_{n}(M=$ $\mathrm{Mn}^{\prime \prime}, 1, \mathrm{Co} ", 2$ and Ni", 3), have been synthesized under hydrothermal conditions. All the compounds were fully characterized by X-ray crystallography, elemental analysis, IR spectroscopy, thermal analysis and magnetic studies. The single crystal $X$-ray analyses show that the $1 \mathrm{D}$ chains of $\left[\mathrm{M}\left(\mathrm{COO}^{-}\right)_{2}\right]_{n}\left(\mathrm{M}=\mathrm{Mn}{ }^{\prime \prime}, 1\right.$, $\mathrm{Co}^{\prime \prime}, 2$ and $\mathrm{Ni}$ ", 3) are further joined by tetrazolyl spacers to form a 3D network. Based on the topological perspective, the complexes can be simplified into a $(3,6)$-connected 2 -nodal net with $\left(4^{2} \cdot 6\right)_{2}\left(4^{4} \cdot 6^{2} \cdot 8^{8} \cdot 10\right)$ "ant" topology. The magnetic measurements reveal different magnetic exchange-coupling interactions: antiferromagnetic for 1 and ferromagnetic for 2 and 3 . Furthermore, the magnetic measurements confirm that spin glass behaviour occurs in 3 but not in 2 .
\end{abstract}

\section{Introduction}

Metal-organic complexes have been attracting considerable attention in the field of crystal engineering owing to their intriguing architectures and topologies and potential applications. ${ }^{1-8}$ Molecular magnetic materials are an important part of the research concerning the application of metal-organic complexes and to date, we have attained impressive advancement in their synthesis, design and characterization. These materials are of great theoretical interest in fields such as quantum tunnelling of magnetization (QTM) and potential technological applications such as qubits for quantum computing etc. ${ }^{9-12}$ However, the design and preparation of magnetic materials such as single molecular magnets (SMMs) or single-chain magnets (SCMs) with bulk magnetic behaviors or special magnetic phenomena are still a challenge. So far, researchers have placed particular emphasis not only on designing new SMMs or SCMs, but also on exploiting other unique magnetic phenomena and behaviors. The selection of metals and ligands for the magnetic materials is extremely important. Usually, the preparation of magnetic materials is focused on the use of paramagnetic metal and bridging ligands

${ }^{a}$ Key Laboratory of Medicinal Chemistry for Natural Resource Education Ministry, School of Chemical Science and Technology Pharmacy, Yunnan University, Kunming, 650091 P. R. China. E-mail: lindu@ynu.edu.cn; qhzhao@ynu.edu.cn; Fax: +86-8715032929; Tel: +86-871-5032929

${ }^{b}$ Department of Chemistry, Northwest University, Xi'an, Shaanxi, 710069 P. R. China $\dagger$ Electronic supplementary information (ESI) available: TGA and IR spectra. CCDC: 725886 for 1, 725885 for 2, 725887 for 3. For ESI and crystallographic data in CIF or other electronic format see DOI: $10.1039 / \mathrm{c} 7 \mathrm{ra07997d}$

$\$$ These authors contributed equally to this paper. because of their abilities in efficient transfer of magnetic exchange interactions. ${ }^{13,14}$ Therefore, the proper choice of ligands is considerably important and it may provide us with the new structures and types as desired. $1 H$-Tetrazolyl-acetic acid (TAA) is a good candidate as it exhibits a variety of coordination abilities and diverse coordination modes. ${ }^{15-21}$ Bai and colleagues reported a 3D mixed-valence iron complex based on the TAA ligand, which shows spin-crossover behaviour. ${ }^{15}$ The Liu research group reported an $\mathrm{Mn}(\mathrm{II})$ compound $\left\{\left[\mathrm{Mn}(\mathrm{TAA})_{2}(-\right.\right.$ $\left.\mathrm{HTAA})_{2}\right] \cdot 2 \mathrm{H}_{2} \mathrm{O}$ \} grounded on TAA, and the magnetic data displayed that a weak AF interaction exists between the two Mn centers. ${ }^{22}$

In addition, 3D isomorphous organic-inorganic hybrid frameworks with diverse magnetic properties haven been relatively rarely reported in literature. The Kitagawa group ${ }^{23}$ reported two isomorphous 3D networks of $\mathrm{Mn}$ (II) and $\mathrm{Co}(\mathrm{II})$ based on naphthalene-1,4-dicarboxylic acid (1,4-napdc $\left.\mathrm{H}_{2}\right)$, showing metamagnetic behaviour $\left(T_{\mathrm{C}}=5.5 \mathrm{~K}\right)$ for $\mathrm{Co}(\mathrm{II})$ and a weak antiferromagnetic interaction for Mn(II). Two isomorphous 3D complexes based on $\mathrm{Co}^{\mathrm{II}} / \mathrm{Ni}^{\mathrm{II}}$, with the $\mathrm{Co}^{\mathrm{II}}$ system exhibiting spin-canted antiferromagnetism were reported by $\mathrm{Bu}$ and coworkers. ${ }^{24}$ Other examples based on the lanthanide(III) state with diverse magnetic properties were reported. ${ }^{25,26}$ Herein, we report three isostructural coordination compounds of the type $\left[\mathrm{M}(\mathrm{TAA})_{2}\right]_{n}$ based on TAA ligand and three different metal ions: $\mathrm{Mn}^{\mathrm{II}}$ for $\mathbf{1}, \mathrm{Co}^{\mathrm{II}}$ for 2 and $\mathrm{Ni}^{\mathrm{II}}$ for 3 , in which the metal ions are bridged via the carboxyl groups to give an eight-membered ring infinite chain with pillared $\mu$-1-tetrazolyl groups generating a 4,6-connected 3D structure. These compounds were synthesized under hydrothermal conditions. The magnetic susceptibility properties of all complexes were measured, and the 
alternating current (ac) magnetic susceptibility measurements of $\mathbf{2}$ and $\mathbf{3}$ were carried out. In addition, thermal stabilities and the IR spectra of the complexes were studied.

\section{Experimental}

\subsection{Materials and methods}

All chemicals were commercially available and used without further purification. Elemental analysis $(\mathrm{C}, \mathrm{H}, \mathrm{N})$ was performed using an ElementarVario ELIII analyzer. Magnetic susceptibility data of the crystalline samples were obtained using a Quantum Design MPMS-XLSQUID magnetometer. The variabletemperature susceptibility measurements were performed in the temperature range of $2-300 \mathrm{~K}$ under an applied field of 1000 Oe. Alternating current (ac) susceptibility measurements were performed with a 2.5 Oe ac oscillating field in an operating frequency range of $10-997 \mathrm{~Hz}$ under a dc field of 0 or 1000 Oe. Thermogravimetric analysis (TGA) data were collected using a simultaneous SDT thermal analyzer at a heating rate of $10{ }^{\circ} \mathrm{C} \min ^{-1}$ under a $\mathrm{N}_{2}$ atmosphere $\left(\mathrm{N}_{2}\right.$ flow rate $=0.06$ $\mathrm{L} \mathrm{min}^{-1}$ ). Fourier transform infrared (FT-IR) spectra were recorded with a Nicolet iS10 Near/Mid-IR Std spectrophotometer using the pressed $\mathrm{KBr}$ pellet in the range of $400-4000 \mathrm{~cm}^{-1}$.

\subsection{Synthesis of complex 1}

A mixture of $\mathrm{MnCl}_{2} \cdot 4 \mathrm{H}_{2} \mathrm{O}(0.2 \mathrm{mmol})$ and TAA $(0.4 \mathrm{mmol})$ was dissolved in $10 \mathrm{~mL}$ of acetonitrile, and the mixture was sealed in a Teflon-lined autoclave and heated at $130{ }^{\circ} \mathrm{C}$ for 3 days. Moccasin-block crystals were collected and washed sequentially by acetonitrile. Yield: $\sim 55 \%$ based on TAA. Anal. calcd (\%) for $\mathrm{C}_{6} \mathrm{H}_{6} \mathrm{MnN}_{8} \mathrm{O}_{4}$ (309.13): $\mathrm{C}, 23.31 ; \mathrm{H}, 1.96 ; \mathrm{N}, 36.25$. Found (\%): C, 22.94; H, 2.32; N, 35.70 .

\subsection{Synthesis of complex 2}

The synthesis method used for compound 2 was similar to that of 1 except that $\mathrm{MnCl}_{2} \cdot 4 \mathrm{H}_{2} \mathrm{O}$ was used instead of $\mathrm{Co}\left(\mathrm{NO}_{3}\right)_{2}-$ $\cdot 6 \mathrm{H}_{2} \mathrm{O}$. Orange red-block crystals were collected and washed sequentially with acetonitrile. Yield: $\sim 75 \%$ based on TAA. Anal. calcd (\%) for $\mathrm{C}_{6} \mathrm{H}_{6} \mathrm{CoN}_{8} \mathrm{O}_{4}$ (313.12): $\mathrm{C}, 23.02 ; \mathrm{H}, 1.93 ; \mathrm{N}, 35.79$. Found (\%): C, 22.81; H, 2.23; N, 35.02.

\subsection{Synthesis of complex 3}

The synthesis method for compound $\mathbf{3}$ is similar to that of $\mathbf{1}$ except that $\mathrm{MnCl}_{2} \cdot 4 \mathrm{H}_{2} \mathrm{O}$ was used instead of $\mathrm{Ni}\left(\mathrm{NO}_{3}\right)_{2} \cdot 6 \mathrm{H}_{2} \mathrm{O}$. Spring green-block crystals were collected and washed sequentially with acetonitrile. Yield: $\sim 79 \%$ based on TAA. Anal. calcd (\%) for $\mathrm{C}_{6} \mathrm{H}_{6} \mathrm{NiN}_{8} \mathrm{O}_{4}$ (312.90): C, 23.03; H, 1.93; N, 35.82. Found (\%): C, 22.97; H, 2.19; N, 35.06.

\subsection{Crystallographic data collection and refinement}

The selected suitable single-crystal was used for the X-ray diffraction analyses. Crystallographic data were collected with graphite-monochromated Mo-K $\alpha$ radiation $(\lambda=0.71073 \AA)$ on Bruker Smart APEX II CCD at $293 \mathrm{~K}$. Empirical absorption corrections were carried out using the SADABS program..$^{27}$ The structures were solved using direct methods and then refined on $F^{2}$ by the full-matrix least-squares technique using SHELXL97 software. ${ }^{28,29}$ All of the non-hydrogen atoms were located using difference Fourier syntheses and were then refined with anisotropic displacement parameters. All the hydrogen atoms of the organic ligands were located using geometrical considerations and isotropically refined with fixed $U$ values using a riding model. Crystallographic data and refinement parameters for complexes 1-3 are summarized in Table 1. The main bond lengths and angles are presented in Tables S1-S3.†

\section{Results and discussion}

\subsection{Crystal structure analysis}

$\mathrm{X}$-ray diffraction analysis indicates that the three compounds are isomorphous with the reported $\mathrm{Zn} / \mathrm{Cd}$ compounds. ${ }^{19,29}$ They crystallized in the monoclinic space group $C 2 / c$. Herein, we only describe the crystal structure of $\mathbf{1}$ in detail as an example.

The asymmetric unit of $\mathbf{1}$ is crystallographically independent with one Mn(II) and one TAA ligand. As shown in Fig. 1a, the $\mathrm{Mn}^{\mathrm{II}}$ ions localize the two fold axis and the crossing point of the mirror plane, which exhibits a distorted octahedral geometry, completed by the four $\mathrm{O}$ atoms from the carboxyl groups in the equatorial plane and two tetrazolyl $\mathrm{N}$ atoms at axial positions with $\mathrm{Mn}-\mathrm{O}$ distances slightly shorter than the $\mathrm{Mn}-\mathrm{N}$ distances. Each TAA ligand acts as a tridentate- $\mathrm{N}, \mathrm{O}, \mathrm{O}^{\prime}$ mode ligand coordinated to three $\mathrm{Mn}^{\mathrm{II}}$ ions through two $1,1-\mu_{2}$ carboxyl oxygen atoms bridging the intrachain $\mathrm{Mn}^{\mathrm{II}}$ ions to form a 1D chain (Fig. 1b) and the tetrazolyl nitrogen atom linking the third $\mathrm{Mn}^{\mathrm{II}}$ ions in the adjacent chain. The $\mathrm{Mn}^{\mathrm{II}}$ ions are spanned via two syn-anti carboxylate oxygen atoms simultaneously composed of $\left[\mathrm{Mn}_{2}\left(\mathrm{COO}^{-}\right)_{2}\right]$ 8-membered ring chain structure with $\mathrm{Mn} \cdots$ Mn distances of $4.865 \AA$ ( $4.786 \AA$ for 2 and $4.785 \AA$ for 3 , respectively). The dihedral angle between the two tetrazolyl rings of the TAA ligands coordinated to the same $\mathrm{Mn}^{\mathrm{II}}$ ions is $79.3^{\circ}\left(80.67^{\circ}\right.$ for 2 and $80.41^{\circ}$ for 3 ), while the two tetrazolyl rings coordinated to the adjacent bridged $\mathrm{Mn}^{\mathrm{II}}$ ions are parallel with each other. Such $\left[\mathrm{Mn}_{2}\left(\mathrm{COO}^{-}\right)_{2}\right]$ 8-member ring chains are further pillared via tetrazolyl spacers to form a 3D network, where the shortest $\mathrm{Mn} \cdots \mathrm{Mn}$ interchain separation is $7.451 \AA$ (7.371 $\AA$ for 2 and $7.314 \AA$ for 3 ) (Fig. 1c). To better understand the structure, topological analysis was undertaken. The $\mathrm{Mn}^{\mathrm{II}}$ ions can be simplified as a 6-connected node and the TAA ligand can be regarded as a 3-connected vertex, which link three nodes to form a $(3,6)$-connected "ant" network with the point symbol of $\left(4^{2} \cdot 6\right)_{2}\left(4^{4} \cdot 6^{2} \cdot 8^{8} \cdot 10\right)$ (Fig. 1d). It is a seldom reported topological net and the reported center material $(\mathrm{Zn} / \mathrm{Cd})$ is not the same. ${ }^{20,22}$

It is worth nothing that the three are isomers reported for complexes 1 and 2 by Dong. ${ }^{20}$ Comparing complex $\mathbf{1} / \mathbf{2}$ and iso-1/ 2 indicates remarkable differences, which results in visible structures and different space groups. The difference between the complexes can be attributed to the discrimination of the $\mathrm{N}-\mathrm{M}-\mathrm{O}$ angles $\left(\theta_{1}\right)$, the $\mathrm{N}-\mathrm{M}-\mathrm{N}$ angles $\left(\theta_{2}\right)$, the $\mathbf{C}-\mathrm{C}-\mathrm{N}$ angles $\left(\theta_{3}\right)$, the dihedral angle $\left(\theta_{4}\right)$ between the plane $\mathrm{A}$ and $\mathrm{B}$, the dihedral angle $\left(\theta_{5}\right)$ between the two tetrazolyl planes and the modes of the two TAA ligands linked by the central metal 
Table 1 Crystal data for $1-3$

\begin{tabular}{|c|c|c|c|}
\hline Compound & 1 & 2 & 3 \\
\hline Empirical formula & $\mathrm{C}_{6} \mathrm{H}_{6} \mathrm{MnN}_{8} \mathrm{O}_{4}$ & $\mathrm{C}_{6} \mathrm{H}_{6} \mathrm{CoN}_{8} \mathrm{O}_{4}$ & $\mathrm{C}_{6} \mathrm{H}_{6} \mathrm{NiN}_{8} \mathrm{O}_{4}$ \\
\hline$F_{\mathrm{w}}$ & 309.13 & 313.12 & 312.90 \\
\hline Crystal system & Monoclinic & Monoclinic & Monoclinic \\
\hline Space group & $C 2 / c$ & $C 2 / c$ & $C 2 / c$ \\
\hline$a / \AA$ & $14.7220(15)$ & $14.4184(16)$ & $14.285(2)$ \\
\hline$b / \AA ̊ ̊$ & $8.4723(9)$ & $8.3825(8)$ & $8.3073(14)$ \\
\hline$c / \AA$ & $9.6326(10)$ & $9.4836(9)$ & $9.4700(16)$ \\
\hline$\beta\left(^{\circ}\right)$ & $119.0620(10)$ & $118.5310(10)$ & $118.178(2)$ \\
\hline$V / \AA^{3}$ & $1050.19(19)$ & $1007.01(18)$ & $990.6(3)$ \\
\hline$Z$ & 4 & 4 & 4 \\
\hline$D_{\mathrm{c}} / \mathrm{g} \mathrm{cm}^{-3}$ & 1.955 & 2.065 & 1.991 \\
\hline $\begin{array}{l}2 \theta \text { range for data } \\
\text { collection }\end{array}$ & 5.76 to 56.52 & 5.82 to 56.58 & 5.88 to 56.66 \\
\hline$\mu / \mathrm{mm}^{-1}$ & 1.287 & 1.736 & 1.287 \\
\hline Reflections collected & 3286 & 4191 & 2986 \\
\hline Unique reflections & 1174 & 1174 & 1150 \\
\hline$F(000)$ & 620.0 & 628.0 & 632.0 \\
\hline$R_{\text {int }}$ & 0.0165 & 0.0165 & 0.0165 \\
\hline GOF on $F^{2}$ & 1.001 & 1.002 & 0.997 \\
\hline$R_{1}{ }^{a}, \mathrm{w} R_{2}^{b}[I>2 \sigma(I)]$ & $0.0234,0.0634$ & $0.0230,0.0617$ & $0.0271,0.0694$ \\
\hline$R_{1}^{a}, \mathrm{w} R_{2}^{b}$ [all data] & $0.0243,0.0641$ & $0.0236,0.0623$ & $0.0319,0.0727$ \\
\hline
\end{tabular}
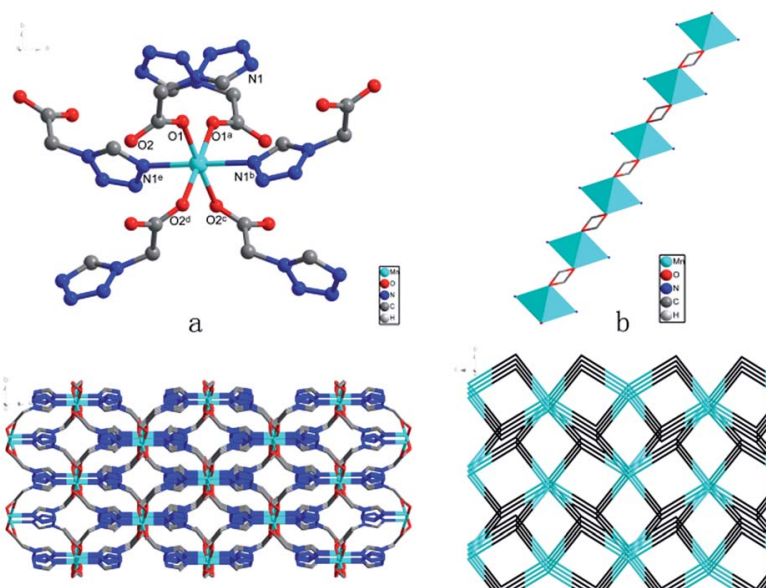

C

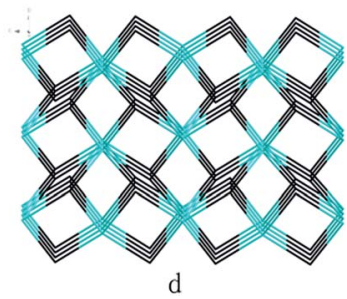

Fig. 1 (a) Coordination environment of $\mathrm{Mn}(\mathrm{II})$ ion in complex 1 with hydrogen atoms omitted for clarity (symmetry codes: (a) $2-x, y, 1.5-$ $z$; (b) $2.5-x, 0.5-y, 2-z$; (c) $x,-y,-0.5+z$; (d) $2-x,-y, 2-z$; (e) $\left.-0.5+x, 0.5-y_{1}-0.5+z\right)$. (b) The $1 \mathrm{D}$ chain of $\left[\mathrm{Mn}\left(\mathrm{COO}^{-}\right)\right]_{n}$. (c) The 3D network of complex 1 down the $c$ axis. (d) View of simplified network in the complex 1 structure with the point symbol $\left(4^{2} \cdot 6\right)_{2}\left(4^{4} \cdot 6^{2} \cdot 8^{8} \cdot 10\right)$.

(Scheme 1). The angles in Table $\mathrm{S} 4 \uparrow$ indicate that the atoms $\mathrm{N}$, $\mathrm{M}, \mathrm{N}$ of the iso- $\mathbf{1} / \mathbf{2}$ are almost on a line; the $\mathrm{N}-\mathrm{M}-\mathrm{N}$ angles $\theta_{2}$ in complex $1 / 2$ are $179.45(6)^{\circ}$ and $179.11(6)^{\circ}$. The $\mathrm{N}-\mathrm{M}-\mathrm{N}$ angles $\theta_{2}$ are nearly the same as the difference values are about $0.3^{\circ}$ for complex 1 and $0.2^{\circ}$ for complex 2. The difference is very apparent when the dihedral angles $\theta_{4}$ and $\theta_{5}$ are contrasted with the angles $\theta_{2}$ and $\theta_{3}$. The dihedral angles $\theta_{4}$ between the plane $\mathrm{A}$ and $\mathrm{B}$ are $84.486(32)^{\circ}$ for 1 and $86.176(34)^{\circ}$ for 2 , and the angles of $\theta_{4}$ are $73.202(29)^{\circ}$ in iso- 1 and $76.814(46)^{\circ}$ in iso-2. The

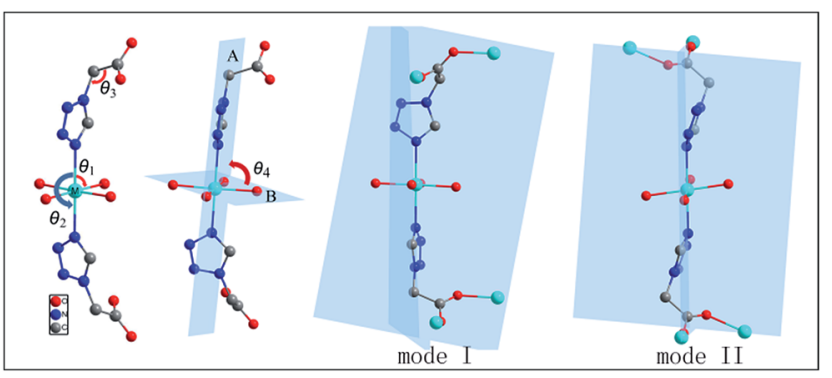

Scheme 1 The four $N-M-O$ angles $\theta_{1}, N-M-N$ angles $\theta_{2}, C-C-N$ angles $\theta_{3}$, dihedral angle between planes (A) and (B) and modes of two TAA ligands linked by central metal.

dihedral angles $\left(\theta_{5}\right)$ between the two tetrazolyl planes are $79.307(53)^{\circ}$ in complex 1 and $80.667(45)^{\circ}$ in complex 2, and the two tetrazolyl planes are parallel in iso-1 and $\mathbf{2}$. The metal atoms are linked by the TAA ligand in a similar syn-anti tridentate coordination mode; in contrast, the modes of the two TAA ligands linked by the central metal are different, which is mode I: syn-syn coordination in complexes $\mathbf{1}$ and 2, and mode II: synanti coordination in iso-1 and 2.

\subsection{Magnetic properties}

Magnetic susceptibility data were collected on powdered crystalline samples of the complexes 1, 2 and 3 at an applied magnetic field of $1 \mathrm{kOe}$ in the temperature range of 2-300 K, which are shown as $\chi_{M} T$ and $1 / \chi_{M}$ versus $T$ plots in Fig. 2 for 1 , Fig. 3 for 2 and Fig. 4 for 3.

For 1 , the $\chi_{\mathrm{M}} T$ value at $300 \mathrm{~K}$ is equal to $4.10 \mathrm{~cm}^{3} \mathrm{~mol}^{-1} \mathrm{~K}$, which is compatible with the expected spin $\operatorname{Mn}^{\mathrm{II}}(S=5 / 2, g=2$, $4.37 \mathrm{~cm}^{3} \mathrm{kmol}^{-1}$ ) anions. With the decrease in temperature, the $\chi_{\mathrm{M}} T$ decreases slowly to $45 \mathrm{~K}$ and then decreases sharply to $2 \mathrm{~K}$. In the temperature range of 5-300 K, the data can be fitted to the Curie-Weiss law $\chi_{\mathrm{M}}=C /(T-\theta)$ expression with a Curie constant $C=4.311 \mathrm{~cm}^{3} \mathrm{~mol}^{-1} \mathrm{~K}$ and the Weiss constant $\theta=$ $-9.47 \mathrm{~K}$, which further suggests the existence of an overall antiferromagnetic exchange interaction between $\mathrm{Mn}^{\mathrm{II}}$ ions.

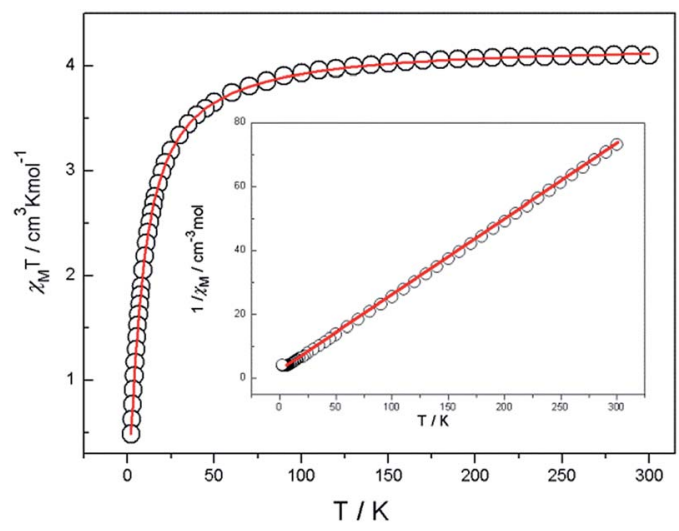

Fig. 2 Temperature dependence of the magnetic susceptibility for complex 1 and $1 / \chi_{M}$ vs. T curve for complex 1 . The red line represents the best-fit curves. 


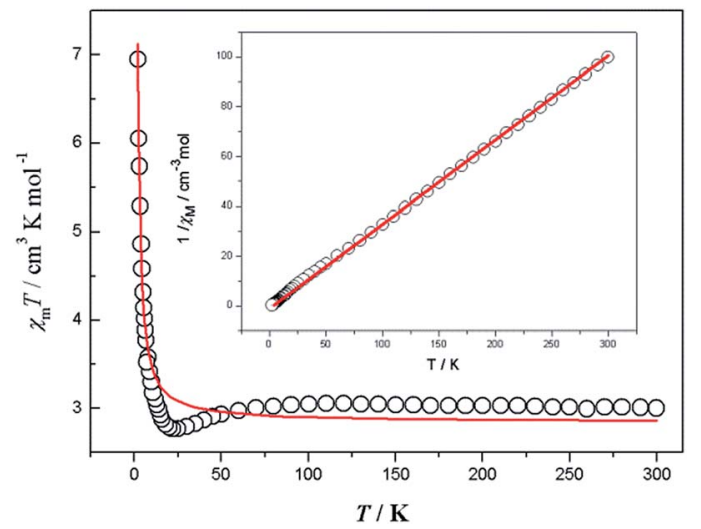

Fig. 3 Temperature dependence of the magnetic susceptibility for complex 2 and $1 / \chi_{M}$ vs. T curve for complex 2 . The red line represents the best-fit curves.

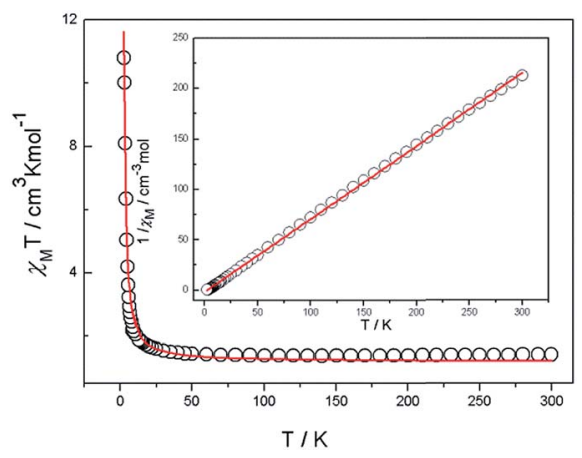

Fig. 4 Temperature dependence of the magnetic susceptibility for complex 3 and $1 / \chi_{M}$ vs. T curve for complex 3 . The red line represents the best-fit curves.

In the case of 2 , the value of $4.56 \mathrm{~cm}^{3} \mathrm{~mol}^{-1} \mathrm{~K} \chi_{\mathrm{M}} T$ at room temperature exceeds the spin only value of $3.75 \mathrm{~cm}^{3} \mathrm{~mol}^{-1} \mathrm{~K}$ as expected for two uncoupled high-spin $\mathrm{Co}^{\mathrm{II}}(S=3 / 2)$ ions with $g=2$. Thus, it indicates that an important orbital contribution exists due to the distorted octahedral ions of $\mathrm{Co}^{\mathrm{II}}$. The $\chi_{\mathrm{M}} T$ first decreases smoothly to a rounded minimum of $2.76 \mathrm{~cm}^{3} \mathrm{~mol}^{-1} \mathrm{~K}$ at $25 \mathrm{~K}$ along with the possible antiferromagnetic interactions between the $\mathrm{Co}^{\mathrm{II}}$ ions. At lower temperatures the $\chi_{\mathrm{M}} T$ plot rises rapidly to a maximum of $6.94 \mathrm{~cm}^{3} \mathrm{~mol}^{-1} \mathrm{~K}$ at $2 \mathrm{~K}$. The magnetic data above $25 \mathrm{~K}$ obey the Curie-Weiss law with $C=3.097$ and a high Weiss constant $\theta$ of $-1.97 \mathrm{~K}$, which further suggest the existence of an overall ferromagnetic exchange interaction between $\mathrm{Co}^{\mathrm{II}}$ ions.

For complex 3, the $\chi_{\mathrm{M}} T$ value is equal to $1.40 \mathrm{~cm}^{3} \mathrm{~mol}^{-1} \mathrm{~K}$ at room temperature. Overall, the $\chi_{\mathrm{M}} T$ value slowly increases with decreasing temperature from $300 \mathrm{~K}$ to $20 \mathrm{~K}$. However, a dramatic increase in the value to $10.79 \mathrm{~cm}^{3} \mathrm{~mol}^{-1} \mathrm{~K}$ at $2 \mathrm{~K}$ was observed. The magnetic behavior of the complex 3 follows the Curie-Weiss law in the entire range of temperature with Curie constants and Weiss temperatures of $1.38 \mathrm{~cm}^{3} \mathrm{~mol}^{-1} \mathrm{~K}$ and 2.57 $\mathrm{K}$, respectively. It suggests ferromagnetic exchange interactions.

According to the structural features of the complexes, the magnetic interaction between metal(II) ions can be estimated by simple chain models, and the mean-field, $z j^{\prime}$, is also introduced. The simplified equation ${ }^{30}$ is as follows:

$$
\begin{gathered}
\chi^{\prime}=\frac{N g^{2} \beta^{2} S(S+1)}{3 k T} \frac{1-\mu}{1+\mu} \\
\mu=\operatorname{coth}\left[\frac{2 J S(S+1)}{k T}\right]-\frac{k T}{2 J S(S+1)} \\
\chi_{\mathrm{M}}=\chi^{\prime} /\left[1-\left(\frac{z j^{\prime} \chi^{\prime}}{N g^{2} x \beta^{2}}\right)\right]
\end{gathered}
$$

where the values of $S$ are 5/2 for complex 1,3/2 for complex 2, and 1 for complex 3 . The best fits to the experimental data give $g=1.96, J=-0.79 \mathrm{~cm}^{-1}, z j^{\prime}=-0.025 \mathrm{~cm}^{-1}$, and $R=1.3 \times 10^{-5}$ for complex 1, $g=2.46, J=0.57 \mathrm{~cm}^{-1}, z j^{\prime}=-0.025 \mathrm{~cm}^{-1}$, and $R=1.3 \times 10^{-5}$ for complex 2 , and $g=2.52, J=4.97 \mathrm{~cm}^{-1}, z j^{\prime}=$ $0.15 \mathrm{~cm}^{-1}$, and $R=1.3 \times 10^{-5}$ for complex 3 ( $R$ value is defined as $\left.\Sigma\left[\left(\chi_{M}\right)_{\text {obs }}-\left(\chi_{M}\right)_{\text {calcd }}\right]^{2} / \Sigma\left(\chi_{M}\right)_{\text {obs }}{ }^{2}\right)$. The fitting results confirm that weak antiferromagnetic coupling exists in 1 , and weak ferromagnetic coupling exists in $\mathbf{2}$ and $\mathbf{3}$.

In order to confirm the existence of special magnetic behavior in complexes $\mathbf{2}$ and $\mathbf{3}$, alternating current (ac) magnetic susceptibility measurements were carried out (Fig. S5 for 2 and $\mathrm{S} 6 \dagger$ for 3). However, there was no frequency dependence for both the in-phase $\left(\chi^{\prime}{ }_{M}\right)$ and out-of phase $\left(\chi^{\prime \prime}{ }_{M}\right)$ susceptibility signals of complex 2. Furthermore, the ac susceptibility measurements of $\mathbf{3}$ were performed in the temperature range of 1.80-2.95 $\mathrm{K}$ under a zero static field oscillating at various frequencies $(10-997 \mathrm{~Hz})$. As revealed in Fig. 5, both in-phase $\left(\chi_{M}^{\prime}\right)$ and out-of phase $\left(\chi^{\prime \prime}{ }_{M}\right)$ susceptibility signals were obviously frequency dependent with a maximum between 2.0-2.2 $\mathrm{K}$ and $1.8-2.0 \mathrm{~K}$, respectively. This was accompanied by an increase in peak temperature as well as a decrease in peak height of $\chi_{{ }_{M}}^{\prime}$ with increasing temperature, while the maximum of the $\chi^{\prime \prime}{ }_{\mathbf{M}}$ peaks shifted to the lower temperature region with decreasing frequencies. Such a shift in temperature of the maximum of $\chi_{{ }_{M}}^{\prime}$ and $\chi^{\prime \prime}{ }_{M}$ with frequency is the positive signature of single molecule magnets (SMMs),

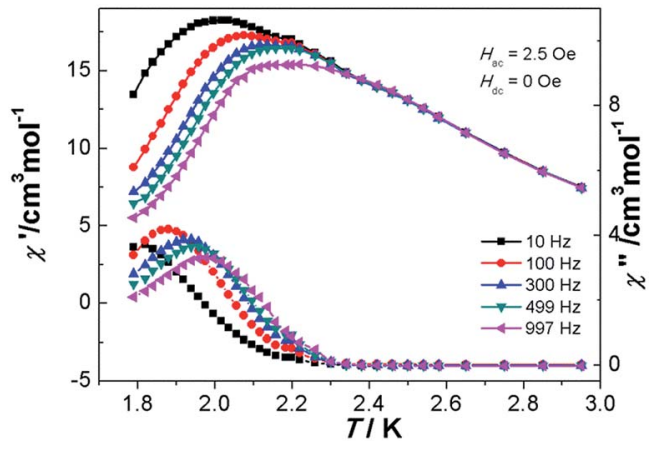

Fig. 5 Temperature dependence of the in-phase $\left(\chi^{\prime}\right)$ and out-ofphase $\left(\chi^{\prime \prime}\right)$ ac susceptibilities in the range of $10-997 \mathrm{~Hz}$ at $H_{\mathrm{ac}}=2.5 \mathrm{Oe}$ for complex 3 measured without an applied dc field. 
single chain magnets (SCMs), and spin glass or a superparamagnetic behavior. ${ }^{31-33}$ The frequency dependence is measured by a parameter $\phi=\left(\Delta T_{\mathrm{p}} / T_{\mathrm{p}}\right) / \Delta(\log f)=0.06$, in which $T_{\mathrm{p}}$ is the temperature at which $\chi^{\prime}$ reaches a maximum. This is the typical value for spin-glass magnets $(\phi<0.1) .{ }^{33}$ In order to gain insight into the real magnetic behavior of 3 , we first tried to determine the relaxation time. The relaxation time $\tau(T)$ data obtained from the $\chi^{\prime \prime}{ }_{\text {м }}$ peaks follow the Arrhenius equation $\tau=$ $\tau_{\mathrm{o}} \exp (\Delta \tau / T)$, resulting in the relaxation time $\tau_{\mathrm{o}}=2.3 \times 10^{-25} \mathrm{~s}$ and the energy barrier $\Delta \tau=94.7 \mathrm{~K}$ (Fig. 6). The rather small value of $\tau_{\mathrm{o}}$ is obtained beyond the normal range of typical SMMs or SCMs $\left(10^{-7}-10^{-12} \mathrm{~s}\right),{ }^{34}$ locating in the usual range for spinglass dynamics in case of $3 .^{33}$ Moreover, another quantitative measurement in a spin-glass system is also determined by fitting the frequency dependence maxima in $\chi^{\prime \prime}{ }_{M}$ using the conventional critical scaling law of the spin dynamics, $\tau=\tau_{\mathrm{o}}\left[\left(T_{\mathrm{p}}\right.\right.$ $\left.\left.-T_{\mathrm{f}}\right) / T_{\mathrm{f}}\right]^{-z v}\left(T_{f}\right.$ and $z v$ represent the freezing temperature and the critical exponent, respectively), ${ }^{35,36}$ with $\tau_{\mathrm{o}}=5.1 \times 10^{-8} \mathrm{~s}, z \nu$ $=9.4$, and $T_{\mathrm{f}}=1.5 \mathrm{~K}$ (Fig. 7). The obtained $z \nu$ value just falls in the typical range (4-12) of the conventional spin glasses. ${ }^{32,33}$ As shown above, all ac measurements and the deduced results are indicative of the canonical spin glass characteristic for 3, which is ascribed to be a ferromagnetic-like layer with spin-glass

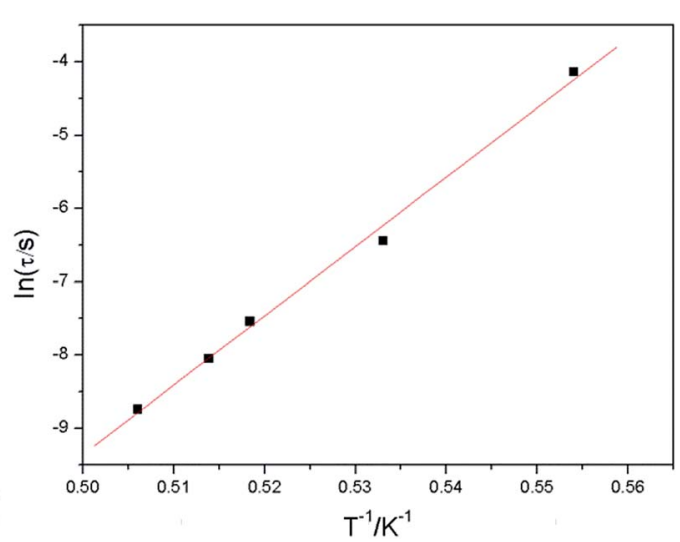

Fig. 6 Plots of $\ln \tau$ vs. $T^{-1}$ of complex 3 .

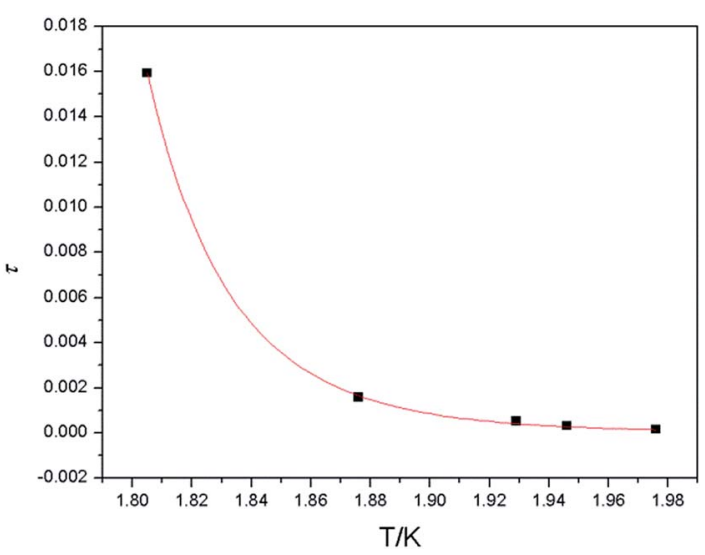

Fig. 7 Temperature dependence of the relaxation time of 3 . dynamic relaxation. Although slow relaxation has been observed in SMMs, SCMs and some magnetic frustration systems, three-dimensional compounds comprising ferromagnetic chains/layers and showing slow magnetic relaxation are very scarce. ${ }^{36-39}$

\subsection{FT-IR spectra and thermal stability}

The IR spectra of the complexes are shown in Fig. S7. $\dagger$ The thermogravimetric analysis (TGA), as shown in Fig. S8, $\dagger$ was performed for complexes 1-3 to examine their stability. Caution: the complexes are easy to explode when heated. Complex 1 remains stable up to $c a .285^{\circ} \mathrm{C}$, finally leading to the formation of the stoichiometric amount of $\mathrm{MnO}_{2}$ as a residue. For complexes 2 and 3 , the framework structure began to decompose form $270{ }^{\circ} \mathrm{C}$ and $200{ }^{\circ} \mathrm{C}$, respectively.

\section{Conclusions}

We used a newly developed tetrazolate carboxylic acid ligand as the bridging ligand in three isomorphous $3 \mathrm{D} \mathrm{Mn}{ }^{\mathrm{II}}, \mathrm{Co}^{\mathrm{II}}$ and $\mathrm{Ni}^{\mathrm{II}}$ coordination polymers and they were observed to possess totally different magnetic properties. Framework 1 shows simple antiferromagnetic interactions. Framework 2 combines canted antiferromagnetic exchange, whereas framework 3 shows spin glass behavior between the $\mathrm{Ni}^{\mathrm{II}}$ ions bridged by the syn-anti carboxyl. Herein, we provide evidence for the existence of diversity in magnetic properties of three isomorphous frameworks with a simple difference of metal ions and thereby demonstrate the importance of engineering metal-organic coordination polymers in the field of molecular-based magnetic materials.

\section{Conflicts of interest}

There are no conflicts to declare.

\section{Acknowledgements}

This research was supported financially by the National Natural Science Foundation of China (No. 21461029, 21561033) and the Natural Science Foundation of YunNan province (Project 2016FB023).

\section{Notes and references}

1 X. Y. Dong, B. Li, B. B. Ma, S. J. Li, M. M. Dong, Y. Y. Zhu, S. Q. Zang, Y. Song, H. W. Hou and T. C. Mak, J. Am. Chem. Soc., 2013, 135, 10214-10217.

2 D. N. Dybtsev, H. Chun, S. H. Yoon, D. Kim and K. Kim, J. Am. Chem. Soc., 2004, 126, 32-33.

3 O. M. Yaghi, M. O'Keeffe, N. W. Ockwig, H. K. Chae, M. Eddaoudi and J. Kim, Nature, 2003, 423, 705-714.

4 Y. Kondo, K. Kon-i, A. Iwasaki, T. Ooi and K. Maruoka, Angew. Chem., Int. Ed., 2000, 39, 414-416.

5 Y. W. Zhao, F. Q. Zhang and X. M. Zhang, ACS Appl. Mater. Interfaces, 2016, 8, 24123-24130. 
6 P. Verma, K. D. Vogiatzis, N. Planas, J. Borycz, D. J. Xiao, J. R. Long, L. Gagliardi and D. G. Truhlar, J. Am. Chem. Soc., 2015, 137, 5770-5781.

7 K.-J. Chen, R.-B. Lin, P.-Q. Liao, C.-T. He, J.-B. Lin, W. Xue, Y.-B. Zhang, J.-P. Zhang and X.-M. Chen, Cryst. Growth Des., 2013, 13, 2118-2123.

8 Z. Z. Lu, R. Zhang, Y. Z. Li, Z. J. Guo and H. G. Zheng, J. Am. Chem. Soc., 2011, 133, 4172-4174.

9 Y. Tian, W. Wang, Y. Chai, J. Cong, S. Shen, L. Yan, S. Wang, X. Han and Y. Sun, Phys. Rev. Lett., 2014, 112, 017202.

10 Y. Dong, P. Yan, X. Zou, T. Liu and G. Li, J. Mater. Chem. C, 2015, 3, 4407-4415.

11 T. N. Nguyen, W. Wernsdorfer, M. Shiddiq, K. A. Abboud, S. Hill and G. Christou, Chem. Sci., 2016, 7, 1156-1173.

12 K. R. Vignesh, S. K. Langley, K. S. Murray and G. Rajaraman, Chem.-Eur. J., 2017, 23, 1654-1666.

13 A. K. Boudalis, B. Donnadieu, V. Nastopoulos, J. M. Clemente-Juan, A. Mari, Y. Sanakis, J.-P. Tuchagues and S. P. Perlepes, Angew. Chem., Int. Ed., 2004, 43, 22662270.

14 T. Gong, X. Lou, J.-J. Fang, E.-Q. Gao and B. Hu, Dalton Trans., 2016, 45, 19109-19116.

15 Y. L. Bai, J. Tao, R. B. Huang, L. S. Zheng, S. L. Zheng, K. Oshida and Y. Einaga, Chem. Commun., 2008, 1753-1755.

16 Y.-B. Lu, S. Jin, F.-M. Jian, Y.-R. Xie and G.-T. Luo, J. Mol. Struct., 2014, 1061, 14-18.

17 X.-Q. Zhang, Q. Yu, H.-D. Bian, X.-G. Bao and H. Liang, J. Coord. Chem., 2009, 62, 2108-2117.

18 D. Ding, M. Zhang, L. Li, Y. Fan and H. Hou, J. Coord. Chem., 2009, 62, 2675-2681.

19 Q. Yu, X. Zhang, H. Bian, H. Liang, B. Zhao, S. Yan and D. Liao, Cryst. Growth Des., 2008, 8, 1140-1146.

20 W.-W. Dong, J. Zhao and L. Xu, Cryst. Growth Des., 2008, 8, 2882-2886.

21 W.-W. Dong, J. Zhao and L. Xu, J. Solid State Chem., 2008, 181, 1149-1154.
22 D.-S. Liu, W.-T. Chen, Y.-P. Xu, P. Shen, S.-J. Hu and Y. Sui, J. Solid State Chem., 2015, 226, 186-191.

23 T. K. Maji, W. Kaneko, M. Ohba and S. Kitagawa, Chem. Commun., 2005, 4613-4615.

24 J. R. Li, Q. Yu, Y. Tao, X. H. Bu, J. Ribas and S. R. Batten, Chem. Commun., 2007, 2290-2292.

25 Y. L. Wang, Y. L. Jiang, Z. J. Xiahou, J. H. Fu and Q. Y. Liu, Dalton Trans., 2012, 41, 11428-11437.

26 X.-W. Liu, R. Guo, H. Liu, Y.-Q. Yu, X.-W. Qi, J.-Y. Xu and C.-Z. Xie, RSC Adv., 2015, 5, 15059-15068.

27 G. M. Sheldrick, Program for Empirical Absorption Correction of Area Detector Data, Germany, 1996.

28 G. M. Sheldrick, SHELX-97, University of Göttingen, Germany, 1997.

29 G. M. Sheldrick, SHELX-97, University of Göttingen, Germany, 1997.

30 A. Das, G. M. Rosair, M. S. El Fallah, J. Ribas and S. Mitra, Inorg. Chem., 2006, 45, 3301-3306.

31 Z. X. Wang, X. L. Li, T. W. Wang, Y. Z. Li, S. Ohkoshi, K. Hashimoto, Y. Song and X. Z. You, Inorg. Chem., 2007, 46, 10990-10995.

32 D. Li, L. Zheng, Y. Zhang, J. Huang, S. Gao and W. Tang, Inorg. Chem., 2003, 42, 6123-6129.

33 J. A. Mydosh, Nurs. Times, 1993, 78, 654.

34 R. Clérac, H. Miyasaka, M. Yamashita and C. Coulon, J. Am. Chem. Soc., 2002, 124, 12837-12844.

35 Y. Z. Zheng, M. L. Tong, W. X. Zhang and X. M. Chen, Angew. Chem., Int. Ed., 2006, 45, 6310-6314.

36 X. J. Li, X. Y. Wang, S. Gao and R. Cao, Inorg. Chem., 2006, 45, 1508-1516.

37 X. N. Cheng, W. X. Zhang, Y. Z. Zheng and X. M. Chen, Chem. Commun., 2006, 3603-3605, DOI: 10.1039/b606765d.

38 X. M. Zhang, P. Li, W. Gao, J. P. Liu and E. Q. Gao, Dalton Trans., 2015, 44, 511-514.

39 J. Liu, J. Luo, Q. Han, J. Cao, L. Chen, Y. Song and J. Zhao, J. Mater. Chem. C, 2017, 5, 2043-2055. 